\title{
A new invariant and parametric connected sum of embeddings
}

\author{
by
}

\section{A. Skopenkov (Moscow)}

Dedicated to the memory of K. Borsuk

\begin{abstract}
We define an isotopy invariant of embeddings $N \rightarrow \mathbb{R}^{m}$ of manifolds into Euclidean space. This invariant together with the $\alpha$-invariant of Haefliger-Wu is complete in the dimension range where the $\alpha$-invariant could be incomplete. We also define parametric connected sum of certain embeddings (analogous to surgery). This allows us to obtain new completeness results for the $\alpha$-invariant and the following estimation of isotopy classes of embeddings. In the piecewise-linear category, for a $(3 n-2 m+2)$-connected $n$ manifold $N$ with $(4 n+5) / 3 \leq m \leq(3 n+2) / 2$, each preimage of the $\alpha$-invariant injects into a quotient of $H_{3 n-2 m+3}(N)$, where the coefficients are $\mathbb{Z}$ for $m-n$ odd and $\mathbb{Z}_{2}$ for $m-n$ even.
\end{abstract}

\section{INTRODUCTION AND MAIN RESULTS}

This paper concerns the classical Knotting Problem: for an $n$-manifold $N$ and a number $m$ describe the set $\mathrm{Emb}^{m}(N)$ of isotopy classes of embeddings $N \rightarrow \mathbb{R}^{m}$. For recent surveys see [ReSk99, Sk07]; whenever possible we refer to these surveys and not to original papers.

All known complete concrete classification results (except for the Haefliger classification of links and smooth knots and recent results [KS05, Sk06, Sk06 ${ }^{\prime}$, CRS07, CRS]) can be obtained using the $\alpha$-invariant of Haefliger-Wu (defined below). For other approaches see [Br68, GW99, CRS04, We].

2000 Mathematics Subject Classification: Primary 57Q35, 57Q37; Secondary 55S15, 55Q91, 57R40.

Key words and phrases: embedding, deleted product, self-intersection, isotopy, Haefliger-Wu invariant.

The author gratefully acknowledges the support by INTAS Grant No. YSF-2002393, the Russian Foundation for Basic Research, Grants No 05-01-00993, 07-01-00648a and 06-01-72551-NCNILa, President of Russian Federation Grants MD-3938.2005.1, MD4729.2007.1 and NSH-4578.2006.1, and by the Pierre Deligne fund based on his 2004 Balzan prize in mathematics. 
We define an isotopy invariant of embeddings which, together with the $\alpha$-invariant, is complete in the dimension range where the $\alpha$-invariant could be incomplete (the $\beta$-Invariant Theorem of $\S 2$ ). We also define parametric connected sum of certain embeddings (see the end of $\S 2$; this is a "surgery" of an embedding preserving the embedded manifold). This leads to new estimations of isotopy classes of embeddings and completeness results for the $\alpha$-invariant (the New Isotopy and Embedding Theorems of $\S 1$ ).

We work in the piecewise linear (PL) category [RS72]. (By [Bry72] for $m \geq n+3$ the classification of embeddings of PL manifolds is the same in the PL and TOP categories. Analogously to $\left[\mathrm{Sk}_{0} 6^{\prime}\right]$ our results give some information for the smooth category.)

Let

$$
\widetilde{N}=\{(x, y) \in N \times N \mid x \neq y\}
$$

be the deleted product of $N$, i.e. the configuration space of ordered pairs of distinct points of $N$. For an embedding $f: N \rightarrow \mathbb{R}^{m}$ one can define a map $\widetilde{f}: \widetilde{N} \rightarrow S^{m-1}$ by the Gauss formula

$$
\widetilde{f}(x, y)=\frac{f x-f y}{|f x-f y|} .
$$

This map is equivariant with respect to the "factors exchange" involution $t(x, y)=(y, x)$ on $\widetilde{N}$ and the antipodal involution on $S^{m-1}$.

Define $\alpha(f)$ to be the equivariant homotopy class of $\tilde{f}$ (cf. [Gr86, 2.1.E]). This is clearly an isotopy invariant.

Let $\pi_{\text {eq }}^{m-1}(\widetilde{N})$ be the set of equivariant maps $\widetilde{N} \rightarrow S^{m-1}$ up to equivariant homotopy. Thus the $\alpha$-invariant is a map

$$
\alpha: \operatorname{Emb}^{m}(N) \rightarrow \pi_{\mathrm{eq}}^{m-1}(\widetilde{N}) .
$$

It is important that using algebraic topology methods one can explicitly calculate the set $\pi_{\mathrm{eq}}^{m-1}(\widetilde{N})$ in many cases [BG71, Bau75, Ya83, ReSk99, Sk02, GS06, Sk07, §5]. So it is very interesting to know under which conditions the $\alpha$-invariant is bijective.

ISOTOPY THEOREM.

(a) [Sk07, the Haefliger-Weber Theorem 5.4] The $\alpha$-invariant is bijective for embeddings $N \rightarrow \mathbb{R}^{m}$ of an $n$-polyhedron $N$ if

$$
2 m \geq 3 n+4 \text {. }
$$

(b) [Sk07, Theorem 5.5] The $\alpha$-invariant is bijective for $m \geq n+3$ and embeddings $N \rightarrow \mathbb{R}^{m}$ of a closed $k$-connected $n$-manifold $N$ if

$$
2 m \geq 3 n+3-k \text {. }
$$

These theorems have many specific corollaries [Sk07].

In this paper we study the case one dimension lower than in the Isotopy Theorem (b). 
By $\mathbb{Z}_{(d)}$ we denote $\mathbb{Z}$ for $d$ even and $\mathbb{Z}_{2}$ for $d$ odd.

New Isotopy Theorem. Let $N$ be a closed $k$-connected orientable n-manifold with

$$
2 m=3 n+2-k \quad \text { and } \quad n \geq 3 k+6 \geq 6 .
$$

(a) The $\alpha$-invariant is surjective and each of its point preimages maps injectively into a certain quotient of $H_{k+1}\left(N ; \mathbb{Z}_{(m-n-1)}\right)$.

(b) The $\alpha$-invariant is bijective if either $(n, k, m)=(6,0,10)$, or $N$ is almost parallelizable and $(n, k, m)=(n, n-14, n+8)$, where $14 \leq$ $n \leq 18$.

The new part of the New Isotopy Theorem is an estimation of point preimages of the $\alpha$-invariant (which is surjective by the Embedding Theorem (b) below). These preimages could a priori be non-trivial by [Sk06 ${ }^{\prime}$, Example 1.6.b] stated below, and could depend on $n, k, N$ and the element of $\pi_{\mathrm{eq}}^{m-1}(\widetilde{N})$ (whose preimage we take).

For $m-n$ even the New Isotopy Theorem (a) implies that these preimages are finite; the orientability assumption can be dropped.

The case $(n, k, m)=(6,0,10)$ of the New Isotopy Theorem (b) shows that [Bau75, Proposition 4] is true in the PL category for 6-manifolds.

Under the assumptions of the New Isotopy Theorem the $\alpha$-invariant is not always injective:

For each even $n \notin\{6,14\}$ and $2 m=3 n+2$ the $\alpha$-invariant is not injective for embeddings $S^{1} \times S^{n-1} \rightarrow \mathbb{R}^{m}$ [Sk06', Example 1.6.b] ( $\left.{ }^{1}\right)$.

Some classification results for $(3 n-2 m+2)$-connected manifold $N=$ $S^{p} \times S^{q}$ are obtained in [Sk06 ${ }^{\prime}$, Theorems 1.3 and 1.4, CRS07, CRS]. It is very surprising that something can be proved for general manifolds $N$.

CONJECTURe.

(a) If $n \geq 3 k+4$ and $N$ is a closed $k$-connected almost parallelizable $n$-manifold, then there is an exact sequence of sets with an action $w$

$$
H_{k+1}\left(N ; \mathbb{Z}_{(m-n-1)}\right) \stackrel{w}{\rightarrow} \operatorname{Emb}^{m}(N) \stackrel{\alpha}{\rightarrow} \pi_{\mathrm{eq}}^{m-1}(\widetilde{N}) \rightarrow 0 .
$$

(b) The Isotopy Theorem (a) holds for $(n, k, m)=(7,1,11)$ and $N$ a spin manifold, as well as for $(n, k, m)=(19,5,27)$ and $N$ almost parallelizable $\left(^{2}\right)$.

The corresponding known and new surjectivity results are as follows $\left({ }^{3}\right)$.

$\left({ }^{1}\right)$ Other examples of non-injectivity of the $\alpha$-invariant are recalled in [ReSk99, 4 , Sk02, §1, Sk07, §5].

$\left({ }^{2}\right)$ This follows from our proof of the Isotopy Theorem (a) (§2) and an improvement $\left[\mathrm{Sk} 06^{\prime}\right.$, Standardization Lemma] of the Standardization Lemma of $\S 2$.

$\left({ }^{3}\right)$ Examples of non-surjectivity of the $\alpha$-invariant are recalled in [ReSk99, §4, Sk07, §5]. 


\section{EMBEDding TheOREM.}

(a) The $\alpha$-invariant is surjective for embeddings $N \rightarrow \mathbb{R}^{m}$ of an $n$ polyhedron $N$ if $2 m \geq 3 n+3$ [Sk07, the Haefliger-Weber Theorem 5.4].

(b) The $\alpha$-invariant is surjective for embeddings $N \rightarrow \mathbb{R}^{m}$ of a closed $k$-connected $n$-manifold $N$ when $2 m \geq 3 n+2-k$ and $m \geq n+3$ [Sk07, Theorem 5.5].

New Embedding Theorem. Let $N$ be a closed $k$-connected $n$-manifold. The manifold $N$ embeds into $\mathbb{R}^{m}$ if there is an equivariant map $\widetilde{N} \rightarrow S^{m-1}$ and either

- $(n, k, m)=(7,0,11)$ and $N$ is orientable, or

- $(n, k, m)=(8,1,12)$ and $N$ is a spin manifold, or

- $(n, k, m)=(n, n-15, n+8)$ and $N$ is almost parallelizable, where $15 \leq n \leq 20$.

An $n$-manifold is p-parallelizable if any embedding $S^{p} \rightarrow N$ can be extended to an embedding $S^{p} \times D^{n-p} \rightarrow N$. Note that 1-parallelizability is equivalent to orientability and $1 \& 2$-parallelizability is equivalent to being a spin manifold $\left({ }^{4}\right)$. The almost parallelizability condition in the results of $\S 1$ can be relaxed to $(k+1)$-parallelizability.

These results were presented at the Borsuk Centenary Conference (Będlewo, 2005) and announced in [Sk05]. I would like to thank M. Skopenkov and S. Melikhov for useful discussions.

\section{PROOFS}

Almost embeddings and almost concordances. An embedding $F: N \times I \rightarrow \mathbb{R}^{m} \times I$ is a concordance if $N \times 0=F^{-1}\left(\mathbb{R}^{m} \times 0\right)$ and $N \times 1=F^{-1}\left(\mathbb{R}^{m} \times 1\right)$. We tacitly use the facts that in codimension at least 3 ,

- concordance implies isotopy [Hu70, Li65],

- every concordance or isotopy is ambient [Hu66, Ak69].

Let $N$ be a connected $n$-manifold and $B^{n} \subset \stackrel{\circ}{N}$ some $n$-ball. The selfintersection set of a map $F: N \rightarrow \mathbb{R}^{m}$ is

$$
\Sigma(F):=\mathrm{Cl}\left\{x \in N \mid \# F^{-1} F x \geq 1\right\} .
$$

A map $F: N \rightarrow \mathbb{R}^{m}$ is an almost embedding of $\left(N, B^{n}\right)$ if $\Sigma(F) \subset B^{n}$, or equivalently, if $\left.F\right|_{N-B^{n}}$ is an embedding and $F\left(N-B^{n}\right) \cap F\left(B^{n}\right)=\emptyset$.

$\left({ }^{4}\right)$ It would be interesting to reformulate the $(k+1)$-parallelizability condition for $k$-connected manifolds in terms of Stiefel-Whitney classes. 
A map $F: N \times I \rightarrow \mathbb{R}^{m} \times I$ is an almost concordance of $\left(N, B^{n}\right)$ if $N \times 0=F^{-1}\left(\mathbb{R}^{m} \times 0\right), \quad N \times 1=F^{-1}\left(\mathbb{R}^{m} \times 1\right) \quad$ and $\quad \Sigma(F) \subset B^{n} \times I$. Instead of the pair $\left(N, B^{n}\right)$ we shall always write simply $N\left({ }^{5}\right)\left({ }^{6}\right)$.

Almost Embedding Theorem. Suppose that $N$ is a closed $k$-connected $n$-manifold, $k \geq 0$ and $m \geq n+2$.

(a) If $f, g: N \rightarrow \mathbb{R}^{m}$ are almost concordant embeddings, then $\alpha(f)=\alpha(g)$ [Sk02, Theorem 5.2. $\alpha$ ].

(b) If $2 m=3 n+2-k$ and $f, g: N \rightarrow \mathbb{R}^{m}$ are embeddings such that $\alpha(f)=\alpha(g)$, then $f$ and $g$ are almost concordant [Sk02, Theorem 2.2.q].

(c) If $2 m=3 n+1-k$ and $\varphi \in \pi_{\mathrm{eq}}^{m-1}(\widetilde{N})$, then there is an almost embedding $F: N \rightarrow \mathbb{R}^{m}$ such that $\alpha(F)=\varphi$ [Sk02, Theorem 2.2.q].

Appendix: some results and conjectures on almost embeddings. This section is not used in the proofs of the main results, but is perhaps of independent interest.

A complete classification of embeddings of a given $n$-manifold $N$ into $S^{n+2}$ up to isotopy (or concordance) seems to be hopeless because it is so for $N=S^{n}$. Therefore it is interesting to obtain a complete classification of embeddings of a given $n$-manifold $N$ into $S^{n+2}$ "modulo knots $S^{n} \rightarrow S^{n+2}$ ". The notion of almost concordance is not only useful to study the initial problem (of classification of embeddings up to concordance) for $m \geq n+3$, but also it is a good notion of "concordance modulo knots $S^{n} \rightarrow S^{n+2}$ ", because any knot $S^{n} \rightarrow S^{n+2}$ is almost concordant to the trivial knot $\left({ }^{7}\right)$ (cf. [MR05]).

We conjecture that almost concordance is equivalent to another natural equivalence relation of "concordance modulo knots $S^{n} \rightarrow S^{n+2}$ ", namely that for a closed $n$-manifold $N$ two embeddings $N \rightarrow S^{n+2}$ are almost concordant if and only if one can be obtained from an embedding concordant to the other by connected summation with knots $S^{n} \rightarrow S^{n+2}$.

$\left({ }^{5}\right)$ Almost embeddings and almost concordances were called quasi-embeddings and quasi-concordances in [Sk02].

$\left.{ }^{6}\right)$ Fix points $x=+1 \in S^{0} \subset S^{p}$ and $y=+1 \in S^{0} \subset S^{n-p}$. By general position for $m \geq n+p+1$ any map $f: S^{p} \times S^{n-p} \rightarrow \mathbb{R}^{m}$ such that $\Sigma(f) \cap x \times S^{n-p}=\emptyset$ is homotopic through such maps to a map $f^{\prime}$ whose self-intersection set is contained in the ball $D_{-}^{p} \times D_{-}^{n-p}$. An analogous statement holds for $m \geq n+p+2$ and almost concordances. In this sense for $m \geq n+p+2$ the above definition of almost concordance for $N=S^{p} \times S^{n-p}$ and $B^{n}=D_{-}^{p} \times D_{-}^{n-p}$ agrees with that of [Sk06 $\left.{ }^{\prime}, \S 2\right]$.

$\left({ }^{7}\right)$ For $N=S^{n_{1}} \sqcup \cdots \sqcup S^{n_{k}}$ the classification of embeddings $N \rightarrow \mathbb{R}^{n+2}$ up to link homotopy is motivated by classification of embeddings $N \rightarrow \mathbb{R}^{n+2}$ "modulo knots $S^{n} \rightarrow \mathbb{R}^{n+2}$. 
Parts (a) and (b) of the following corollary follow from the Almost Embedding Theorem (b) and [Sk02, Theorem 2.3.q], respectively.

Corollary.

(a) Let $N$ be a sphere with $g$ handles. Then the set of PL almost embeddings $N \rightarrow \mathbb{R}^{4}$ up to $P L$ almost concordance is in 1-1 correspondence with $\mathbb{Z}_{2}^{2 g} \cong H_{1}\left(N ; \mathbb{Z}_{2}\right)$.

(b) For a closed simply-connected 4-manifold $N$, the set of smooth almost embeddings $N \rightarrow \mathbb{R}^{6}$ up to $P L$ almost concordance is in 1-1 correspondence with $\pi_{\text {eq }}^{5}(\widetilde{N})$.

We conjecture that the set of PL embeddings $S^{1} \times S^{1} \rightarrow \mathbb{R}^{4}$ up to PL almost concordance consists of exactly three elements (i.e., the almost embedding $S^{1} \times S^{1} \rightarrow \mathbb{R}^{4}$ corresponding by Corollary (a) to the class $(1,1) \in$ $H_{1}\left(S^{1} \times S^{1} ; \mathbb{Z}_{2}\right)$ is not almost concordant to a PL embedding) $\left.{ }^{8}\right)$.

The restriction $k \geq 0$ is essential in the Almost Embedding Theorem (b) $\left({ }^{9}\right)$.

We conjecture that the Almost Embedding Theorem (b) holds in the smooth category, and that in [Sk02, Theorem 2.3.q] and in the injectivity part of [Sk02, Theorem 2.3. $\alpha$ ] we can replace the PL category by DIFF (if $N$ is a smooth manifold) $\left({ }^{10}\right)$.

We conjecture that for $n$ even, $m=(3 n+1-k) / 2 \geq n+3$ and a $k$-connected closed $n$-manifold $N$ such that $H_{k+1}(N)$ is free there is an exact sequence of sets $\operatorname{Emb}^{m}(N) \stackrel{\alpha}{\rightarrow} \pi_{\mathrm{eq}}^{m-1}(\tilde{N}) \stackrel{\beta}{\rightarrow} H_{k+1}(N)$ (cf. the New Embedding Theorem $\left({ }^{11}\right)$ ).

Definition of $\beta$-invariant. The Almost Embedding Theorem (b) suggests the definition of an invariant, required for classification of embeddings when $2 m \leq 3 n+2-k$. For each almost concordance $F$ between embeddings, analogously to [Hu69, XI.4.iii, Hu70', p. 408, Ha84, §1] we define an

$\left(^{8}\right)$ A related result states that any PL embedding $S^{2} \sqcup \cdots \sqcup S^{2} \rightarrow S^{4}$ is link homotopic to the trivial embedding [BT99, Ba01] (the case of two components was proved earlier by Hosokawa-Suzuki).

$\left({ }^{9}\right)$ Indeed, for $l \notin\{3,7\}$ take a link $f: S^{0} \times S^{2 l-1} \rightarrow \mathbb{R}^{3 l}$ such that $\lambda_{12}(f)=$ $\lambda_{21}(f)=\left[\iota_{l}, \iota_{l}\right]$. Then $\alpha(f)=\Sigma^{\infty} \lambda_{21}(f)=0$ but $f$ is not almost concordant to the standard embedding.

$\left({ }^{10}\right)$ This could perhaps be proved analogously to the cited results using the relative version of [Sk02, Disjunction Theorem 3.1].

$\left({ }^{11}\right)$ By [Sk02, Theorem 2.3.q] and the $\beta$-Invariant Theorem it suffices to prove that for an almost embedding $F: N \rightarrow \mathbb{R}^{m}$ the obstruction $\beta(F)$ does not depend on $\left.F\right|_{B^{n}}$ (but only on $\left.F\right|_{N-\dot{B}^{n}}$ ). This obstruction is a map $b: \pi_{n}(M) \rightarrow H_{k+1}(N)$. We can prove that $b$ is constant by checking that $\pi_{n}(M)$ is finite and for fixed $\varphi_{0} \in \pi_{n}(M)$ the map $\varphi \mapsto b(\varphi)-b\left(\varphi_{0}\right)$ is a homomorphism. 
obstruction $\beta(F)$ to modification of $F$ to a concordance. Roughly speaking, $\beta(F)$ measures the linking of $\Sigma(F)$ with $F(N)$.

Analogous invariants are the Sato-Levine invariant of knots, the Hudson-Habegger obstruction to embedding disks and the Fenn-Rolfsen-Koschorke-Kirk $\beta$-invariant of link maps (see references in $\left[\mathrm{Sk}_{06}{ }^{\prime}\right]$ ). In the proof of the New Embedding and Isotopy Theorems we do not use the definition but only use the properties of the $\beta$-invariant (they are stated in the $\beta$-Invariant Theorem of the next subsection).

In this and the next subsections we omit the coefficients $\mathbb{Z}_{(m-n-1)}$ of chain groups in the notation.

Suppose that $N$ is a connected orientable $n$-manifold (possibly with boundary) and $F: N \rightarrow B^{m}$ is a proper general position almost embedding whose restriction to the boundary is an embedding. ( $F$ could be an almost concordance between embeddings.) Take a triangulation $T$ of $N$ such that $B^{n}$ is a subcomplex of $T$ and $F$ is linear on simplices of $T$. Then $\Sigma(F)$ is a subcomplex of $T$. Denote by $[\Sigma(F)] \in C_{2 n-m}\left(B^{n}\right)$ the sum of the top-dimensional simplices of $\Sigma(F)$.

For $m-n$ odd the coefficient \pm 1 of an oriented simplex $\sigma \subset \Sigma(F)$ is defined as follows $\left({ }^{12}\right)$. Fix in advance any orientation of $N$ and of $B^{m}$. By general position there is a unique simplex $\sigma^{\prime}$ of $T$ such that $F(\sigma)=F\left(\sigma^{\prime}\right)$. The orientation on $\sigma$ induces an orientation on $F \sigma$ and then on $\sigma^{\prime}$. The orientations on $\sigma$ and $\sigma^{\prime}$ induce orientations on the normal spaces in $N$ to these simplices. These two orientations (in this order) together with the orientation on $F \sigma$ induce an orientation on $B^{m}$. If this orientation agrees with the fixed orientation of $B^{m}$, then the coefficient of $\sigma$ is +1 , otherwise it is -1 . Clearly, a change of orientation of $\sigma$ changes the sign of $\sigma$ in $[\Sigma(F)]$, so the sign is well-defined $\left({ }^{13}\right)$.

By [Hu69, Lemma 11.4, Hu70', Lemma 1], $\partial[\Sigma(F)]=0\left({ }^{14}\right)$. Hence

$$
[\Sigma(F)]=\partial C \quad \text { for some } C \in C_{2 n-m+1}\left(B^{n}\right) .
$$

By $\left[\mathrm{Hu} 70^{\prime}\right.$, Corollary 1.1], $\partial F C=0$. Hence

$$
F(C)=\partial D \quad \text { for some } D \in C_{2 n-m+2}\left(B^{m}\right) .
$$

\footnotetext{
$\left({ }^{12}\right)$ For $m-n$ even, this sign can also be defined but is not used.

$\left({ }^{13}\right)$ This definition of sign is equivalent to Hudson's given as follows. The orientation on $\sigma$ induces an orientation on $F \sigma$ and on $\sigma^{\prime}$, hence also on their links. Consider the oriented $(2 m-2 n-1)$-sphere $\mathrm{lk} F \sigma$, the link of $F \sigma$ in a triangulation of $B^{m}$, "compatible" with $T$. This sphere contains disjoint oriented $(m-n-1)$-spheres $F\left(\mathrm{lk}_{T} \sigma\right)$ and $F\left(\mathrm{lk}_{T} \sigma^{\prime}\right)$. Their linking coefficient $\operatorname{link}_{\mathrm{lk} F \sigma}\left(F\left(\mathrm{lk}_{T} \sigma\right), F\left(\mathrm{lk}_{T} \sigma^{\prime}\right)\right) \in \mathbb{Z}_{(m-n-1)}$ is the coefficient of $\sigma$ in $[\Sigma(F)]$, which equals \pm 1 .

$\left({ }^{14}\right)$ Here we use the fact that the coefficients are $\mathbb{Z}_{2}$ for $m-n$ even.
} 
By general position

$$
\widetilde{D}:=\left(\left.F\right|_{N-\operatorname{Int} B^{n}}\right)^{-1}(D) \in C_{3 n-2 m+2}(N) .
$$

Since the support of $C$ is in $B^{n}$, the support of $F(C)=\partial D$ is in $F\left(B^{n}\right)$. Hence $\widetilde{D}$ is a cycle and we can define $\left({ }^{15}\right)$

$$
\beta(F):=[\widetilde{D}] \in H_{3 n-2 m+2}\left(N ; \mathbb{Z}_{(m-n-1)}\right) .
$$

Proof that $\beta(F)$ is well-defined, i.e. independent of the choices of $C$ and $D$. The independence of the choice of $D$ is standard. Let us prove the independence of the choice of $C$. For an almost embedding $F$, if $\partial C_{1}=$ $\partial C_{2}=[\Sigma(F)]$ then $\partial\left(C_{1}-C_{2}\right)=0$. Hence

$$
C_{1}-C_{2}=\partial X \quad \text { for some } X \in C_{2 n-m+1}\left(B^{n}\right) \text {. }
$$

Thus $F C_{1}-F C_{2}=\partial F X$. Hence we can take chains $D_{1}$ and $D_{2}$ as above and such that $D_{1}-D_{2}=F X$. Since the support of $X$ is in $B^{n}$, we have $\widetilde{D}_{1}=\widetilde{D}_{2}$.

\section{Properties of the $\beta$-invariant and proof of the New Isotopy Theorem (a)}

$\beta$-InVARIANT THEOREM. Let $N$ be a connected orientable $n$-manifold (possibly with boundary) and $m \geq n+3$. To each proper general position almost embedding $F: N \rightarrow B^{m}$ whose restriction to the boundary is an embedding there corresponds an element

$$
\beta(F) \in H_{3 n-2 m+3}\left(N ; \mathbb{Z}_{(m-n-1)}\right)
$$

with the following properties:

- (Obstruction) If $F$ is an embedding, then $\beta(F)=0$.

- (Invariance) $\beta(F)$ is invariant under almost concordance of $F$ relative to the boundary.

- (Completeness) If $\beta(F)=0$ and $N$ is homologically $(3 n-2 m+1)$ connected, then $F$ is almost concordant $\operatorname{rel}\left(N-\dot{B}^{n}\right)$ to an embedding.

- (Additivity) Suppose that $N=X \times I$ and $F, F^{\prime}$ are almost concordances between $f_{0}$ and $f_{1}, f_{1}$ and $f_{2}$, respectively, Denote by $\bar{F}$ the reversed $F$, i.e. $\bar{F}(x, t)=F(x, 1-t)$. Define an almost concordance $F \cup F^{\prime}$ between $f_{0}$ and $f_{2}$ as the "union" $\left({ }^{16}\right)$ of

$$
F: X \times[0,1] \rightarrow \mathbb{R}^{m} \times[0,1] \text { and } F^{\prime}: X \times[1,2] \rightarrow \mathbb{R}^{m} \times[1,2] .
$$

Then $\beta\left(F \cup F^{\prime}\right)=\beta(F)+\beta\left(F^{\prime}\right)$ and $\beta(\bar{F})=-\beta(F)$.

$\left({ }^{15}\right)$ This definition agrees with that for $N=S^{p} \times S^{q}\left[\mathrm{Sk} 06^{\prime}\right.$, subsection "A new embedding invariant" of $\S 2]$ when $m \geq 2 p+q+2$.

$\left({ }^{16}\right)$ Observe that the union of almost concordances is associative up to ambient isotopy. 
Here the orientability assumption can be dropped for $m-n$ even.

The obstruction and additivity follow obviously from the definition of $\beta$-invariant.

The invariance is analogous to [Hu70', Lemma 2, cf. Hu69, Lemma 11.6]. The completeness is a non-trivial property, but it is an easy consequence of known results $[\mathrm{Hu} 70$ ', Theorem 2, Ha84, Theorem 4]. See the details below.

Proof of the invariance. Let $F_{0}$ and $F_{1}$ be two almost concordances between embeddings $f$ and $g$. Suppose that $\Phi: N \times I \rightarrow B^{m} \times I$ is an almost concordance between $F_{0}$ and $F_{1}$. As in the above definition of $\beta\left(F_{0}\right)$ and $\beta\left(F_{1}\right)$, take chains

$$
C_{0}, C_{1} \in C_{2 n-m+1}\left(B^{n}\right) \quad \text { such that } \partial C_{j}=\left[\Sigma\left(F_{j}\right)\right] \text { for } j \in\{0,1\} .
$$

Analogously to the above definition of $\beta(F)$ define

$$
[\Sigma(\Phi)] \in C_{2 n-m+1}\left(B^{n} \times I\right) .
$$

Let $i_{j}: N \cong N \times j \rightarrow N \times I$ be the inclusions. Then

$$
\partial[\Sigma(\Phi)]=i_{1}\left[\Sigma\left(F_{1}\right)\right]-i_{0}\left[\Sigma\left(F_{0}\right)\right], \quad \text { so } \quad \partial\left([\Sigma(\Phi)]+C_{0}-C_{1}\right)=0 .
$$

Therefore there exists

$$
C \in C_{2 n-m+2}\left(B^{n} \times I\right) \text { such that } \partial C=[\Sigma(\Phi)]+C_{1}-C_{0} .
$$

Analogously to $\left[\mathrm{Hu} 70^{\prime}\right.$, Lemma 2$], \partial \Phi C=i_{1}\left(F_{1} C_{1}\right)-i_{0}\left(F_{0} C_{0}\right)$. Let pr : $N \times I \rightarrow N$ be the projection. Hence $\partial \operatorname{pr} \Phi C=F_{1} C_{1}-F_{0} C_{0}$. Thus analogously to the proof of the independence of $\beta$ from the choice of $C$ we obtain $\beta\left(F_{0}\right)=\beta\left(F_{1}\right)$.

Proof of the completeness. Write

$$
M:=B^{m}-\operatorname{Int} R_{B^{m}}\left(F\left(N-\stackrel{\circ}{B}^{n}\right), F \partial B^{n}\right) .
$$

Observe that $\left.F\right|_{B^{n}}: B^{n} \rightarrow M$ is a proper map whose restriction to the boundary is an embedding.

Consider the following composition of Alexander and Poincaré duality isomorphisms (with $\mathbb{Z}$ coefficients):

$$
\begin{aligned}
H_{i}(M) & \cong H^{m-i-1}\left(B^{m}-M, \partial B^{m}-M\right) \cong H^{m-i-1}\left(N-\stackrel{\circ}{B}^{n}, \partial N\right) \\
& \cong H_{i+n-m+1}\left(N-\stackrel{\circ}{n}^{n}, \partial B^{n}\right) \cong H_{i+n-m+1}(N) .
\end{aligned}
$$

Since $N$ is homologically $(3 n-2 m+1)$-connected, $M$ is homologically $(2 n-m)$-connected. Since $M$ is simply-connected, it follows that it is $(2 n-m)$-connected. Then by $\left[\mathrm{Hu}^{\prime} 0^{\prime}\right.$, Theorem 2, Ha84, Theorem 4] the class $[F C] \in H_{2 n-m+1}\left(M ; \mathbb{Z}_{(m-n-1)}\right)$ is the complete obstruction to the 
existence of a homotopy rel $\partial B^{n}$ from $\left.F\right|_{B^{n}}: B^{n} \rightarrow M$ to an embedding. This class goes to $\beta(F)=0$ under the composition of the above isomorphisms with $\mathbb{Z}_{(m-n-1)}$ coefficients. Hence $\left.F\right|_{B^{n}}$ is homotopic rel $\partial B^{n}$ to an embedding. Extending this embedding over $N$ by $F$ we obtain the required embedding $N \rightarrow B^{m}$.

Proof of the New Isotopy Theorem (a). Fix any $\varphi \in \pi_{\mathrm{eq}}^{m-1}(\tilde{N})$ and any embedding $f_{0}: N \rightarrow \mathbb{R}^{m}$ such that $\alpha\left(f_{0}\right)=\varphi$. Define

$$
\begin{aligned}
K:=\left\{\beta\left(F_{0}\right) \in H_{k+1}(N ;\right. & \left.\mathbb{Z}_{(m-n-1)}\right) \mid \\
& \left.F_{0} \text { is an almost concordance from } f_{0} \text { to } f_{0}\right\} .
\end{aligned}
$$

By the additivity of the $\beta$-invariant, $K$ is a subgroup (depending on $n, k$, $N, \varphi)$.

For any embedding $f: N \rightarrow \mathbb{R}^{m}$ such that $\alpha(f)=\alpha\left(f_{0}\right)$, by the Almost Embedding Theorem (b) there is an almost concordance $F$ from $f$ to $f_{0}$. (This together with the additivity of the $\beta$-invariant implies that $K$ does not depend on the choice of $f_{0}$.) So we can define a map

$$
B: \alpha^{-1}(\varphi) \rightarrow H_{k+1}\left(N ; \mathbb{Z}_{(m-n-1)}\right) / K \quad \text { by } \quad B(f):=\beta(F)+K .
$$

If $F$ and $F^{\prime}$ are two almost concordances from $f$ to $f_{0}$, then $F^{\prime} \cup \bar{F}$ is an almost concordance from $f_{0}$ to $f_{0}$. Hence the map $B$ is well-defined by the additivity of the $\beta$-invariant.

If $B(f) \in K$, then $\beta(F)=\beta\left(F_{0}\right)$ for some almost concordance $F_{0}$ from $f_{0}$ to $f_{0}$. Then $F \cup \bar{F}_{0}$ is an almost concordance from $f$ to $f_{0}$, and by the additivity of the $\beta$-invariant, $\beta\left(F \cup \bar{F}_{0}\right)=0$. Hence by the completeness of the $\beta$-invariant, $f$ is concordant to $f_{0}$. Thus $B$ is injective.

Parametric connected sum of embeddings. We denote by

$$
S^{p}=D_{+}^{p} \underset{\partial D_{+}^{p}=S^{p-1}=\partial D_{-}^{p}}{\cup} D_{-}^{p}
$$

the standard decomposition of $S^{p}$. Analogously define $\mathbb{R}_{ \pm}^{m}$ and $\mathbb{R}^{m-1}$. Identify $D^{p}$ with $D_{+}^{p}$.

For $m \geq n+2$ denote by $i$ the standard embedding which is the composition $S^{p} \times S^{n-p} \rightarrow \mathbb{R}^{p+1} \times \mathbb{R}^{n-p+1} \subset \mathbb{R}^{m} \subset S^{m}$.

Let $N$ be a closed connected $n$-manifold. Let $s: S^{p} \times D^{n-p} \rightarrow N$ be an embedding. For the ball $B^{n} \subset N$ from the definition of an almost embedding (concordance), assume that $\operatorname{im} s \cap B^{n}=\emptyset$.

A map $f: N \rightarrow S^{m}$ is called s-standardized if

- $f \circ s: S^{p} \times D^{n-p} \rightarrow D_{-}^{m}$ is the restriction of the standard embedding,

- $f(N-\operatorname{im} s) \subset \operatorname{Int} D_{+}^{m}$.

Roughly speaking, a map $N \rightarrow D^{m}$ is $s$-standardized if its image is put on the hyperplane $D^{m-1}$ so that the image intersects the hyperplane in a 
standardly embedded $S^{p} \times D^{n-p}$ (indeed, for such a map the set im $s$ can be pulled below the hyperplane to obtain an $s$-standardized embedding in the above sense) $\left({ }^{17}\right)$.

A concordance $F: N \times I \rightarrow S^{m} \times I$ between $s$-standardized maps is called $s$-standardized if

- $F(\operatorname{im} s \times I) \subset S_{-}^{m} \times I$ is the identical concordance,

- $F((N-\operatorname{im} s) \times I) \subset \operatorname{Int} S_{+}^{m} \times I$.

\section{StANDARDizATion Lemma.}

(a) If $m \geq n+p+2$, then any (almost) embedding $g: N \rightarrow S^{m}$ is isotopic to an s-standardized (almost) embedding.

(b) If $m \geq n+p+3$, then any (almost) concordance between s-standardized embeddings $N \rightarrow S^{m}$ is isotopic relative to the ends to an s-standardized (almost) concordance.

Proof of (a). Fix a point $y \in D_{-}^{n-p} \subset S^{n-p}$. Since $m \geq n+p+2 \geq$ $2 p+2$, it follows that $\left.g\right|_{S^{p} \times y}$ is unknotted in $S^{m}$. So there is an embedding $\widehat{g}: D^{p+1} \rightarrow S^{m}$ such that

$$
\left.\widehat{g}\right|_{\partial D^{p+1}}=\left.g\right|_{S^{p} \times y} \quad \text { and } \quad \widehat{g} \operatorname{Int} D^{p+1} \cap g N=\emptyset .
$$

(The second property holds by general position because $m \geq n+p+2$.) The regular neighborhood in $S^{m}$ of $\widehat{g} D^{p+1}$ is homeomorphic to the $m$-ball. Take an isotopy moving this ball to $D_{-}^{m}$ and let $f^{\prime}: N \rightarrow S^{m}$ be the embedding obtained from $g$.

Now we are done since the embedding $f^{\prime} \circ s$ is isotopic to the standard embedding by the following result (because $m \geq n+3$, the pair $\left(S^{p} \times D^{n-p}, S^{p} \times S^{n-p-1}\right)$ is $(n-p-1)$-connected and $\left.n-p-1 \geq 2 n-m+1\right)$.

Unknotting Theorem Moving the Boundary. Let $N$ be a compact $n$-dimensional $P L$ manifold and $f, g: N \rightarrow D^{m}$ proper $P L$ embeddings. If $m \geq n+3$ and $(N, \partial N)$ is $(2 n-m+1)$-connected, then $f$ and $g$ are properly isotopic [Hu69, Theorem 10.2, p. 199].

Proof of (b). This is a relative version of the proof of (a). Take a concordance $G$ between standardized embeddings $f_{0}, f_{1}: N \rightarrow S^{m}$. There is a level-preserving embedding $\widehat{G}: D^{p+1} \times\{0,1\} \rightarrow S^{m} \times\{0,1\}$ whose components satisfy $(*)$. Since $m+1 \geq p+1+3$, by the Haefliger-Zeeman Unknotting Theorem any concordance $S^{p} \times I \rightarrow S^{m} \times I$ standard on the

$\left({ }^{17}\right)$ Note that standardized in the sense of $\left[\mathrm{Sk} 06^{\prime}, \S 2\right]$ is $i$-standardized in the sense of this paper. 
boundary is isotopic to the standard concordance. Hence the map $\widehat{G}$ can be extended to an embedding $\widehat{G}: D^{p+1} \times I \subset S^{m} \times I$ such that

$$
\left.\widehat{G}\right|_{\partial D^{p+1} \times I}=\left.G\right|_{S^{p} \times y \times I} \text { and } \widehat{G}\left(\operatorname{Int} D^{p+1} \times I\right) \cap G(N \times I)=\emptyset .
$$

(The second property holds by general position because $m \geq n+p+3$.) Take a regular neighborhood $B^{m} \times I$ in $S^{m} \times I$ of $\widehat{G} D^{p+1}$ such that

$$
\left(B^{m} \times I\right) \cap\left(S^{m} \times\{0,1\}\right)=D_{-}^{m} \times\{0,1\} .
$$

Take an isotopy of $S^{m} \times I \operatorname{rel} S^{m} \times\{0,1\}$ moving $B^{m} \times I$ to $D_{-}^{m} \times I$. Let $F^{\prime}$ be the concordance obtained from $G$ by this isotopy.

The embedding $\left.F^{\prime}\right|_{\operatorname{im} s \times I}: \operatorname{im} s \times I \rightarrow D_{-}^{m} \times I$ is isotopic rel $D_{-}^{m} \times\{0,1\}$ to the identical concordance by the following Unknotting Theorem Moving Part of the Boundary (which is proved analogously to [Hu69, Theorem 10.2 on p. 199]).

Let $N$ be a compact $n$-dimensional PL manifold, $A$ a codimension zero submanifold of $\partial N$ and $f, g: N \rightarrow D^{m}$ proper PL embeddings. If $m \geq n+3$ and $(N, A)$ is $(2 n-m+1)$-connected, then $f$ and $g$ are properly isotopic $\operatorname{rel} \partial N-A$.

Denote by $R_{k}$ the symmetry of $\mathbb{R}^{k}$ with respect to the plane $x_{1}=x_{2}=0$.

Definition (of parametric connected sum). (a) Let $f: N \rightarrow \mathbb{R}^{m}$ and $g: S^{p} \times S^{n-p} \rightarrow \mathbb{R}^{m}$ be (almost) embeddings. If $m \geq n+p+2$, then by Standardization Lemma (a) we can make isotopies and assume that $f$ and $g$ are $s$-standardized and $i$-standardized, respectively. Define an (almost) embedding

$$
f \#_{s} g: N \rightarrow \mathbb{R}^{m} \quad \text { by } \quad\left(f \#_{s} g\right)(a)= \begin{cases}f(a), & a \notin \operatorname{im} s, \\ R_{m} g\left(x, R_{n-p} y\right), & a=s(x, y) .\end{cases}
$$

(b) Take (almost) concordances

$$
F: N \times I \rightarrow \mathbb{R}^{m} \times I \text { and } G: S^{p} \times S^{n-p} \times I \rightarrow \mathbb{R}^{m} \times I .
$$

If $m \geq n+p+3$, then by the Standardization Lemma (b) we can make isotopies relative to the ends and assume that $F$ and $G$ are $s$-standardized and $i$-standardized, respectively. Define an (almost) concordance $F \#_{s} G$ : $N \times I \rightarrow \mathbb{R}^{m} \times I$ by

$$
\left(F \#_{s} G\right)(a, t)= \begin{cases}F(a, t), & a \notin \operatorname{im} s, \\ \left(R_{m} G\left(x, R_{n-p} y, t\right), t\right), & a=s(x, y) .\end{cases}
$$

We do not need parametric connected sum to be independent of the choice of an almost concordance to a standardized almost embedding or almost concordance: we denote by $f \#_{s} g$ or $F \#_{s} G$ the result for any such choice. 


\section{Proof of the New Isotopy Theorem (b) and the New Embedding Theorem}

The Hopf Invariant Lemma. Take the standard embedding $i$ : $D^{p+1} \times S^{q} \rightarrow S^{m}$. Represent $\varphi \in \pi_{p+q}\left(S^{m-q-1}\right.$ ) by a map (not necessarily an embedding)

$$
\bar{\varphi}: S^{p+q} \rightarrow S^{m}-i\left(D^{p+1} \times S^{q}\right) \simeq S^{m-q-1} .
$$

If $2 m=3 q+2 p+2$, then $\beta(i \# \bar{\varphi})= \pm H \Sigma \varphi\left({ }^{18}\right)$.

Proof. We argue analogously to [Ko88, Theorem 4.8]. We may assume that $\bar{\varphi}$ is a smooth general position framed immersion. Extend $\bar{\varphi}$ to a smooth general position framed immersion $\widehat{\varphi}: B \rightarrow \mathbb{R}^{m}$, where $B:=B^{p+q+1}$. Then by [Ko88, Theorem 1.3] and [Ke59, Lemma 5.1] the class $\pm \Sigma \varphi \in \pi_{p+2 q+1-m}^{S}$ is represented by the framed $(p+2 q+1-m)$-submanifold

$$
\Delta:=\left\{(u, z) \in B \times S^{q} \mid \widehat{\varphi}(u)=i(a, z)\right\} \quad \text { of } \quad B \times S^{q} \subset S^{p+2 q+1-m}
$$

(with natural framing). For a 0 -chain $X$ with coefficients in $\mathbb{Z}_{(m-p-q-1)}$ in a connected manifold denote by $[X]$ the number of points in $X$ modulo 2 when $m-p-q$ is even (we need only this case for the Non-Triviality Lemma) and the algebraic number of points when $m-p-q$ is odd (That is, $[X]$ is the 0-dimensional homology class of $X$.) Then by [Ko88, p. 411],

$$
\pm H \Sigma \varphi=\left[\left\{(x, y) \in \Delta \times \Delta \mid \operatorname{pr}_{2} x=\operatorname{pr}_{2} y\right\}\right]
$$

(this set is finite by general position). Thus

$$
\begin{aligned}
\pm H \Sigma \varphi & =\left[\left\{(u, v, z) \in B \times B \times S^{q} \mid \widehat{\varphi}(u)=\widehat{\varphi}(v)=i(a, z)\right\}\right] \\
& =\left[i\left(a \times S^{q}\right) \cap \widehat{\varphi} \operatorname{pr}_{2} D\right]=\beta(i \# \bar{\varphi}),
\end{aligned}
$$

where $D:=\{(u, v) \in B \times B \mid \widehat{\varphi}(u)=\widehat{\varphi}(v)\}$. Here the last equality holds because

$$
C:=\{(u, v) \in \partial B \times B \mid \bar{\varphi}(u)=\widehat{\varphi}(v)\}, \quad D \quad \text { and } \quad \widetilde{D}:=\widehat{\varphi} \operatorname{pr}_{2} D
$$

are as in the definition of the $\beta$-invariant ( $C$ has the natural orientation for $m-p-q$ odd $)$; the groups $H_{p}\left(S^{p} \times S^{q} ; \mathbb{Z}_{(m-p-q-1)}\right)$ and $\mathbb{Z}_{(m-p-q-1)}$ are identified by the isomorphism $\gamma \mapsto \gamma \cap\left[a \times S^{q}\right]$. The definition of $\beta$-invariant does make sense in the piecewise-smooth category (and hence in the smooth category). Recall that the piecewise-smooth category is equivalent to the PL category, i.e. the forgetful map from the set of PL embeddings up to $\mathrm{PL}$ isotopy to the set of piecewise differentiable embeddings up to piecewise differentiable isotopy is a 1-1 correspondence [Hae67].

Non-Triviality Lemma. For $1 \leq p<l \in\{3,7\}$ there exists an almost embedding $G: S^{p} \times S^{2 l} \rightarrow \mathbb{R}^{3 l+p+1}$ such that $\beta(G)=1$.

$\left({ }^{18}\right)$ Here $i \# \bar{\varphi}$ is the embedded connected sum of linked embeddings but not the parametrized connected sum as above; $i \# \bar{\varphi}=\bar{\mu}(\varphi)$ in the notation of [Sk06 $\left.{ }^{\prime}\right]$. 
Proof. Since $p \geq 1$, the group $\pi_{2 l+p}\left(S^{l+p}\right)$ is either stable or metastable, so the stable suspension $\Sigma^{\infty}$ is epimorphic. Since $l \in\{3,7\}$, the Hopf invariant $H$ is epimorphic. Hence there is $\varphi \in \pi_{2 l+p}\left(S^{l+p}\right)$ whose stable Hopf invariant $H \Sigma^{\infty}(\varphi)$ is $1 \in \mathbb{Z}_{2}$. By the Hopf Invariant Lemma for $q=2 l$ and $m=3 l+p+1$ we obtain $\beta(i \# \bar{\varphi})=H \Sigma^{\infty}(\varphi)=1$.

Realization Lemma. Let $N$ be an orientable $(p-1)$-connected $p$ parallelizable closed $n$-manifold and $n \geq 2 p+1$. Then any homology class $x \in H_{p}\left(N ; \mathbb{Z}\right.$ or $\left.\mathbb{Z}_{2}\right)$ is realizable by an embedding $\bar{x}: S^{p} \times D^{n-p} \rightarrow N$.

Proof. Since $N$ is $(p-1)$-connected, any homology class in $H_{p}(N ; \mathbb{Z})$ can be realized by a map $S^{p} \rightarrow N$. Hence the same holds for $\mathbb{Z}_{2}$ coefficients. Since $n \geq 2 p+1$ every such map is homotopic to an embedding $S^{p} \rightarrow N$. Since $N$ is $p$-parallelizable, it follows that this embedding can be extended to an embedding $\bar{x}: S^{p} \times D^{n-p} \rightarrow N$.

\#-Additivity Lemma. If $p=3 n-2 m+3 \geq 0, m \geq n+p+2$ and $s: S^{p} \times D^{n-p} \rightarrow N$ is an embedding, then $\beta\left(f \#_{s} g\right)=\beta(f)+\beta(g)[s]$ for almost embeddings $f: N \rightarrow \mathbb{R}^{m}$ and $g: S^{p} \times S^{n-p} \rightarrow \mathbb{R}^{m}$, where $\beta(g)$ is considered as an element of $\mathbb{Z}_{(m-n-1)}$.

Proof. Since $m \geq n+p+2$, by the Standardization Lemma (a) we may assume that $f$ and $g$ are standardized. Since $R_{m}$ and $R_{n-p}$ are isotopic to the identity maps of $\mathbb{R}^{m}$ and of $S^{n-p}$, they do not change orientations. Hence

$$
\left[\Sigma\left(f \#_{s} g\right)\right]=[\Sigma(f)]+s_{*}\left(\operatorname{id} S^{p} \times R_{n-p}\right)_{*}[\Sigma(g)],
$$

so we can take

$$
C_{f \#_{s} g}:=C_{f}+s_{*}\left(\operatorname{id} S^{p} \times R_{n-p}\right)_{*} C_{g} \text { and } D_{f \#_{s} g}:=D_{f}+R_{m} D_{g} .
$$

We may assume that the supports of $D_{f}$ and $D_{g}$ are in $\mathbb{R}_{+}^{m}$. Identify $S^{p} \times$ $D^{n-p}$ with $S^{p} \times D_{+}^{n-p}$ so that it would contain the support of $\widetilde{D}_{g}$. Then

$$
\begin{aligned}
\beta\left(f \#_{s} g\right) & =\left[\widetilde{D}_{f \#_{s} g}\right] \\
& =\left[\left(\left.F \#_{s} G\right|_{N-\operatorname{Int} B^{n}}\right)^{-1} D_{f}\right]+\left[\left(\left.F \#_{s} G\right|_{N-\operatorname{Int} B^{n}}\right)^{-1} R_{m} D_{g}\right] \\
& =\left[\widetilde{D}_{f}\right]+s_{*}\left[\widetilde{D}_{g}\right]=\beta(f)+[s] \beta(g) .
\end{aligned}
$$

Proof of the New Embedding Theorem. Take any $\varphi \in \pi_{\mathrm{eq}}^{m-1}(\widetilde{N})$. Since $2 m=3 n+1-k$, by the Almost Embedding Theorem (c) there is an almost embedding $f: N \rightarrow \mathbb{R}^{m}$ such that $\alpha(f)=\varphi$. By the Realization Lemma there is an embedding $s: S^{k+1} \times D^{n-k-1} \rightarrow N$ such that $[s]=-\beta(f)$. By the Non-Triviality Lemma there is an almost embed$\operatorname{ding} G: S^{k+1} \times S^{n-k-1} \rightarrow \mathbb{R}^{m}$ such that $\beta(G)=1$. By the \#-Additivity Lemma, $\beta\left(f \#_{s} G\right)=\beta(f)+[s]=0$. By the completeness of the $\beta$-invariant, 
$f \#{ }_{s} G$ is almost concordant to an embedding $N \rightarrow \mathbb{R}^{m}$. (Note that possibly $\left.\alpha(f) \neq \alpha\left(f \#_{s} G\right).\right)$

Proof of the New Isotopy Theorem (b). Since $2 m=3 n+2-k$, the surjectivity follows by the Embedding Theorem (b). The following proof of the injectivity is a relative version of the proof of the New Embedding Theorem.

Take two embeddings $f, f^{\prime}: N \rightarrow \mathbb{R}^{m}$ such that $\alpha(f)=\alpha\left(f^{\prime}\right)$. By the Almost Embedding Theorem (b) there is an almost concordance $F$ from $f$ to $f^{\prime}$. By the Realization Lemma there is an embedding $s: S^{k+1} \times D^{n-k-1} \rightarrow N$ such that $[s]=-\beta(F)$.

Take an almost embedding $G: S^{k+1} \times S^{n-k} \rightarrow \mathbb{R}^{m+1}$ given by the Non-Triviality Lemma. Analogously to the Standardization Lemma we may assume that

- $G\left(S^{k+1} \times D_{-}^{n-k}\right) \subset D_{-}^{m+1}$ is the standard embedding,

- $G\left(S^{k+1} \times \frac{1}{2} D_{+}^{n-k}\right) \subset \frac{1}{2} D_{+}^{m+1}$ is the standard embedding,

- $G\left(S^{k+1} \times\left(D_{+}^{n-k}-\frac{1}{2} D_{+}^{n-k}\right)\right) \subset D_{+}^{m+1}-\frac{1}{2} D_{+}^{m+1}$.

The last inclusion gives an almost concordance $G_{0}$ between standard embeddings such that $\beta\left(G_{0}\right)=1$. Then $F \#_{s} G_{0}$ is an almost concordance from $f$ to $f^{\prime}$.

Analogously to the \#-Additivity Lemma one proves the following.

If $p=3 n-2 m+2 \geq 0, m \geq n+p+3$ and $s: S^{p} \times D^{n-p} \rightarrow N$ is an embedding, then $\beta\left(F \#_{s} G_{0}\right)=\beta(F)+\beta\left(G_{0}\right)[s]$ for almost concordances $F: N \times I \rightarrow \mathbb{R}^{m} \times I$ and $G_{0}: S^{p} \times S^{n-p} \times I \rightarrow \mathbb{R}^{m} \times I$, where $\beta\left(G_{0}\right)$ is considered as an element of $\mathbb{Z}_{(m-n-1)}$.

So $\beta\left(F \#_{s} G_{0}\right)=\beta(F)+[s]=0$. Hence by the completeness of the $\beta$-invariant, $f$ is isotopic to $f^{\prime}$.

\section{References}

[Ak69] E. Akin, Manifold phenomena in the theory of polyhedra, Trans. Amer. Math. Soc. 143 (1969), 413-473.

[Ba01] A. Bartels, Higher dimensional links are singular slice, Math. Ann. 320 (2001), 547-576.

[BT99] A. Bartels and P. Teichner, All two-dimensional links are null-homotopic, Geom. Topol. 3 (1999), 235-252.

[Bau75] D. R. Bausum, Embeddings and immersions of manifolds in Euclidean space, Trans. Amer. Math. Soc. 213 (1975), 263-303.

[BG71] J. C. Becker and H. H. Glover, Note on the embedding of manifolds in Euclidean space, Proc. Amer. Math. Soc. 27 (1971), 405-410.

[Br68] W. Browder, Embedding smooth manifolds, in: Proc. Int. Congress Math. (Moscow, 1966), Mir, Moscow, 1968, 712-719. 
[Bry72] J. L. Bryant, Approximating embeddings of polyhedra in codimension 3, Trans. Amer. Math. Soc. 170 (1972), 85-95.

[CRS04] M. Cencelj, D. Repovš and A. Skopenkov, On the Browder-Levine-Novikov embedding theorems, Trudy Mat. Inst. Steklova 247 (2004), 280-290 (in Russian); English transl.: Proc. Steklov Inst. Math. 247 (2004), 259-268.

[CRS07] M. Cencelj, D. Repovš and M. Skopenkov, Homotopy type of the complement of an immersion and classification of embeddings of tori, Uspekhi Mat. Nauk 62 (2007), no. 5, 165-166 (in Russian); English transl.: Russian Math. Surveys $62(2007)$.

[CRS] - - - - Knotted tori and the $\beta$-invariant, 2005, preprint.

[GS06] D. Gonçalves and A. Skopenkov, Embeddings of homology equivalent manifolds with boundary, Topology Appl. 153 (2006), 2026-2034.

[GW99] T. Goodwillie and M. Weiss, Embeddings from the point of view of immersion theory, II, Geom. Topol. 3 (1999), 103-118.

[Gr86] M. Gromov, Partial Differential Relations, Ergeb. Math. Grenzgeb. 9, Springer, Berlin, 1986.

[Ha84] N. Habegger, Obstructions to embedding disks II. A proof of a conjecture of Hudson, Topology Appl. 17 (1984), 123-130.

[Hae67] A. Haefliger, Lissage des immersions - I, Topology 6 (1967), 221-240.

[Hu66] J. F. P. Hudson, Extending piecewise linear isotopies, Proc. London Math. Soc. (3) 16 (1966), 651-668.

[Hu69] -, Piecewise-Linear Topology, Benjamin, New York, 1969.

[Hu70] -, Concordance, isotopy and diffeotopy, Ann. of Math. 91 (1970), 425-448.

[Hu70'] - Obstruction to embedding disks, in: Topology of Manifolds (Athens, GA, 1969), Markham, Chicago, IL, 1970, 407-415.

[Ke59] M. Kervaire, An interpretation of $G$. Whitehead's generalization of $H$. Hopf's invariant, Ann. of Math. 69 (1959), 345-362.

[Ko88] U. Koschorke, Link maps and the geometry of their invariants, Manuscripta Math. 61 (1988), 383-415.

[KS05] M. Kreck and A. Skopenkov, A classification of smooth embeddings of 4-manifolds in the 7-space, arXiv:math/0512594.

[Li65] W. B. R. Lickorish, The piecewise linear unknotting of cones, Topology 4 (1965), 67-91.

[MR05] S. Melikhov and D. Repovš, n-Quasi-isotopy: I. Questions of nilpotence, J. Knot Theory Ramif. 14 (2005), 571-602.

[ReSk99] D. Repovš and A. Skopenkov, New results on embeddings of polyhedra and manifolds into Euclidean spaces, Uspekhi Mat. Nauk 54 (1999), no. 6, 61109 (in Russian); English transl.: Russian Math. Surveys 54 (1999), no. 6, 1149-1196.

[RS72] C. P. Rourke and B. J. Sanderson, Introduction to Piecewise-Linear Topology, Ergeb. Math. Grenzgeb. 69, Springer, Berlin, 1972.

[Sk02] A. Skopenkov, On the Haefliger-Hirsch-Wu invariants for embeddings and immersions, Comment. Math. Helv. 77 (2002), 78-124.

[Sk05] -, A new invariant and parametric connected sum of embeddings, arXiv:math/ 0509621.

[Sk06] - A classification of smooth embeddings of 3-manifolds in the 6-space, arXiv:math/0603429; Math. Z., to appear.

[Sk06 $\left.{ }^{\prime}\right] \quad-$, Classification of embeddings below the metastable dimension, arXiv:math/ 0607422 . 
[Sk07] A. Skopenkov, Embedding and knotting of manifolds in Euclidean spaces, in: Surveys in Contemporary Mathematics, N. Young and Y. Choi (eds.), London Math. Soc. Lecture Note. Ser. 347, Cambridge Univ. Press, 2007, 248-342.

[We] M. Weiss, Second and third layers in the calculus of embeddings, preprint.

[Ya83] T. Yasui, On the map defined by regarding embeddings as immersions, Hiroshima Math. J. 13 (1983), 457-476.

Department of Differential Geometry

Faculty of Mechanics and Mathematics

Moscow State University

Moscow 119992, Russia

and

Independent University of Moscow

B. Vlasyevskiy, 11, Moscow 119002, Russia

E-mail: skopenko@mccme.ru

Received 17 October 2005;

in revised form 11 November 2007 